\title{
Probing TeV Physics through Lattice Neutron-Decay Matrix Elements
}

\section{Huey-Wen Lin*}

Department of Physics, University of Washington, Seattle, WA 98195-1560

\section{Tanmoy Bhattacharya}

Los Alamos National Laboratory, T-2, Los Alamos, NM 87545

\section{Saul D. Cohen}

Institude of Nuclear Theory, University of Washington, Seattle, WA 98195-1560

\section{Rajan Gupta}

Los Alamos National Laboratory, T-2, Los Alamos, NM 87545

\section{Anosh Joseph}

Deutsches Elektronen-Synchrotron DESY, Platanenallee 6, 15738 Zeuthen, Germany

\section{Boram Yoon}

Los Alamos National Laboratory, T-2, Los Alamos, NM 87545

\begin{abstract}
Precision low-energy measurements provide constraints on the Standard Model and can discern the signatures predicted for particles beyond the Standard Model. In the case of the neutron beta decay, potential new $\mathrm{TeV}$-scale particles may be probed through scalar and tensor interactions. Lattice QCD provides the most accurate low-energy nucleon matrix elements needed to relate the experiments to BSM constraints. These constraints from the low-energy sector will be compared with current and near-future limits provided by the LHC.
\end{abstract}

2013 International Workshop on Computational Science and Engineering 14-17 October 2013

National Taiwan University, Taipei, Taiwan

\footnotetext{
*Speaker.
} 


\section{Introduction}

The Standard Model (SM) is one of the great successes of modern physics, and all experiments done so far are consistent with the predictions of the SM within reasonable errors. However, there are certain deficiencies of the Standard Model that lead us to consider physics beyond the Standard Model (BSM). The LHC has been built to discover the mechanism for electroweak symmetry breaking and to probe certain potential new BSM particles which appear at the TeV scale, such as some candidates for dark matter. Such high-energy colliders in general are well suited to production of high-mass particles directly at their resonances and for probing processes with cross sections that scale with energy. However, there are many low-energy precision experiments that also have the ability to probe BSM physics. These generally fall into two classes: experiments that can very precisely measure physics that is precisely predicted by the SM, such as muon $g-2$, the proton weak charge, and flavor-changing processes involving the CKM matrix. Another class of experiments can look for tiny signals in places that the SM says should be either undetectably small or exactly zero.

Precision measurements of nucleons fall into this class; they provide constraints on the SM and can discern the signatures predicted for BSM particles at the 1-10-TeV scale. Knowing the SM inputs to nucleon matrix elements will be necessary to constrain the couplings of dark-matter candidates such as the neutralino, to relate the neutron electric dipole moment to the CP-violating theta parameter, or to search for new $\mathrm{TeV}$-scale particles though non- $V-A$ interactions in neutron beta decay.

One such unique and timely experiment is precision measurement of neutron beta-decay parameters at the ultra-cold neutron (UCN) source at Los Alamos National Laboratory (LANL) [1]. The advantage of using UCN is that the helicity-flip factor of $m_{e} / E_{e}$ that makes it difficult to observe chirality-violating BSM corrections at high energies is of order unity in neutron decays, due to the small kinetic energy of the emitted electron. As a result, the signal of interest is of the same size as the SM background in UCN decays.

Using the neutron or nuclear beta decay to discover new particles would be conceptually quite similar to how Fermi theory led to the discovery of the electroweak interaction and its bosons. Beta decay was originally explained by Fermi by adding a new term to the fundamental Lagrangian describing 4-fermion interactions. Such a theory introduces a coupling, the Fermi constant, that has units of inverse-energy squared. This can be interpreted as a coupling constant of order 1 over some energy scale squared. Looking at the value, you can see that this energy scale $\Lambda$ would be around $100 \mathrm{GeV}$. People suspected that when the theory was directly probed in colliders at energies near a hundred $\mathrm{GeV}$, the Fermi theory would break down and some new physics must occur. As it turns out, the Fermi theory is a low-energy effective theory approximating the electroweak theory, which has 3 vector bosons, the $W$ 's and $Z$. The theory was later probed in high-energy proton-antiproton experiments at CERN, and the new particles were found with resonances around the scale predicted by this interpretation of the Fermi theory. We can imagine that new particles beyond the Standard Model can be predicted in just such a way: by first detecting the low-energy effective operators and later directly probing them in a high-energy experiment.

The neutron beta-decay Hamiltonian contains a $V-A$ current from the Standard Model weak 
interaction for the leptons and the quarks, and we add terms corresponding to new BSM physics:

$$
H_{\mathrm{eff}}=G_{F}\left(J_{V-A}^{\mathrm{lept}} \times J_{V-A}^{\mathrm{quark}}+\sum_{i} \varepsilon_{i}^{\mathrm{BSM}} \hat{O}_{i}^{\mathrm{lept}} \times \hat{O}_{i}^{\text {quark }}\right),
$$

where $G_{F}$ is the Fermi constant, $J_{V-A}$ indicates the left-handed current of the indicated particle, and the sum includes operators with novel chiral structure. So in the context of our theory, new operators will enter with the coefficients $\varepsilon$ that are related to the $\mathrm{TeV}$ scale of the particles. The leptonic part is understandable using analytic techniques, but the quark operator in the context of the nucleon will introduce some unknown coupling constants,

$$
g_{T}=\left\langle n\left|\bar{u} \sigma_{\mu v} d\right| p\right\rangle, g_{S}=\langle n|\bar{u} d| p\rangle,
$$

which are nonperturbative functions of the nucleon structure, described in the SM by quantum chromodynamics (QCD). Any deviation from the SM $V-A$ current coming from the new scalar and tensor interactions in the effective theory will require knowledge of the couplings $g_{S}$ and $g_{T}$ to understand.

The knowledge of these coupling from experiment and previous QCD models are rather limited. The tensor charge $g_{T}$ is the zeroth moment of transversity and can be studied through processes such as SIDIS (semi-inclusive deep inelastic scattering). The HERMES and COMPASS experiments [2,3] presented their first estimates of $g_{T}$ from data collected at $Q^{2}=2.4 \mathrm{GeV}^{2}$. Experimentally, to estimate $g_{T}$ one first extracts the contribution of individual quarks as a function of the quark momentum fraction $x$ at a particular $Q^{2}$. To obtain the contribution of each quark, the results, estimated from measurements at a finite number of values of $x$, are integrated over the full range $0 \leq x \leq 1$. The isovector tensor charge is then given by the difference between the up and down quark contributions. Since this analysis requires data over the full range of $x$, and the low- $x$ and high- $x$ values are not well known, improvements in precision await future experiments. Current extracted numbers are highly model-dependent. Combining SIDIS (HERMES and COMPASS) results with the Belle $e^{+} e^{-}$analysis [4, 2] of data collected at $Q^{2}=110 \mathrm{GeV}^{2}$, the best experimental estimate of $g_{T}$ at $Q^{2}=0.8 \mathrm{GeV}^{2}$ is $0.77_{-0.36}^{+0.18}$. There are also estimates from purely theoretical models. These include the Nambu-Jona-Lasinio model [5] and the chiral-quark soliton model [6]; unfortunately, they are not consistent with each other. Estimates from QCD sum rules [7] have a large uncertainty. Overall, model estimates of the tensor charge range within 0.6,2.3 [8].

The nucleon isovector scalar charge $g_{S}$, on the other hand, has no experimental measurements, and theoretical estimates [9] (from different model approximations) give rather loose bounds: $0.25 \leq g_{S} \leq 1$. Given that $g_{S, T}$ are poorly determined in both models and experiment, it would be advantageous to use a theoretical tool, such as lattice QCD, that can give better quantitative control over the uncertainties. We will revisit this in later sections.

Experimentally, two quantities characterizing neutron decay provide high sensitivity to new physics by having small and precisely known Standard-Model background in neutron decay $(n \rightarrow$ $p e \bar{v}_{e}$ ): the Fierz interference term (b), which characterizes the deviation of the energy spectrum of the emitted electrons from leading-order SM predictions, and the energy dependence of the neutrino asymmetry parameter $(B)$ that measures the asymmetry in the momentum of $\bar{v}_{e}$ relative to 
the neutron polarization $\vec{\sigma}_{n}$.

$$
d \Gamma \propto F\left(E_{e}\right)\left[1+a \frac{\vec{p}_{e} \cdot \vec{p}_{v}}{E_{e} E_{v}}+b \frac{m_{e}}{E_{e}}+B \frac{\vec{\sigma}_{n} \cdot \vec{p}_{v}}{E_{v}}+A \frac{\vec{\sigma}_{n} \cdot \vec{p}_{e}}{E_{e}}+\ldots\right],
$$

where $\vec{p}_{e}$ and $\vec{p}_{v}$ are the outgoing electron and neutrino momenta, and $E_{e, v}$ are the electron and neutrino energies. $F\left(E_{e}\right)$ is the tree-level SM electron energy spectrum in the no-recoil limit.

Within the SM, $a$ and $A$ are $O(1)$, whereas $b$ and the energy-dependent component $b_{v}$ in $B=B_{0}+\left(m_{e} / E_{e}\right) b_{v}$ are $O\left(10^{-3}\right)$ and known from theory to the level of $10^{-5}$ [10]. Therefore, deviations from the SM predictions for $b$ and $b_{v}$ at a level larger than $O\left(10^{-5}\right)$ would unambiguously signal the presence of BSM interactions [10,11]. These parameters are much less accessible in high-energy experiments, because the interference of new interactions with the SM amplitudes always involves a spin flip, which is suppressed by the factor $m_{e} / E_{e} \ll 1$, while in neutron decay $m_{e} / E_{e} \sim 1$.

On left-hand side of Fig. 1, we show how precision measurements of $b$ and $b_{v}$ at the $10^{-3}$ level can reveal the existence of new physics with mass scale $\Lambda_{i}$ in the multi-TeV range, which will be explored directly at the LHC. Furthermore, the two panels highlight the difference in bounds between using previous phenomenological estimates for $g_{S}$ and $g_{T}$ and current LQCD estimates. In these
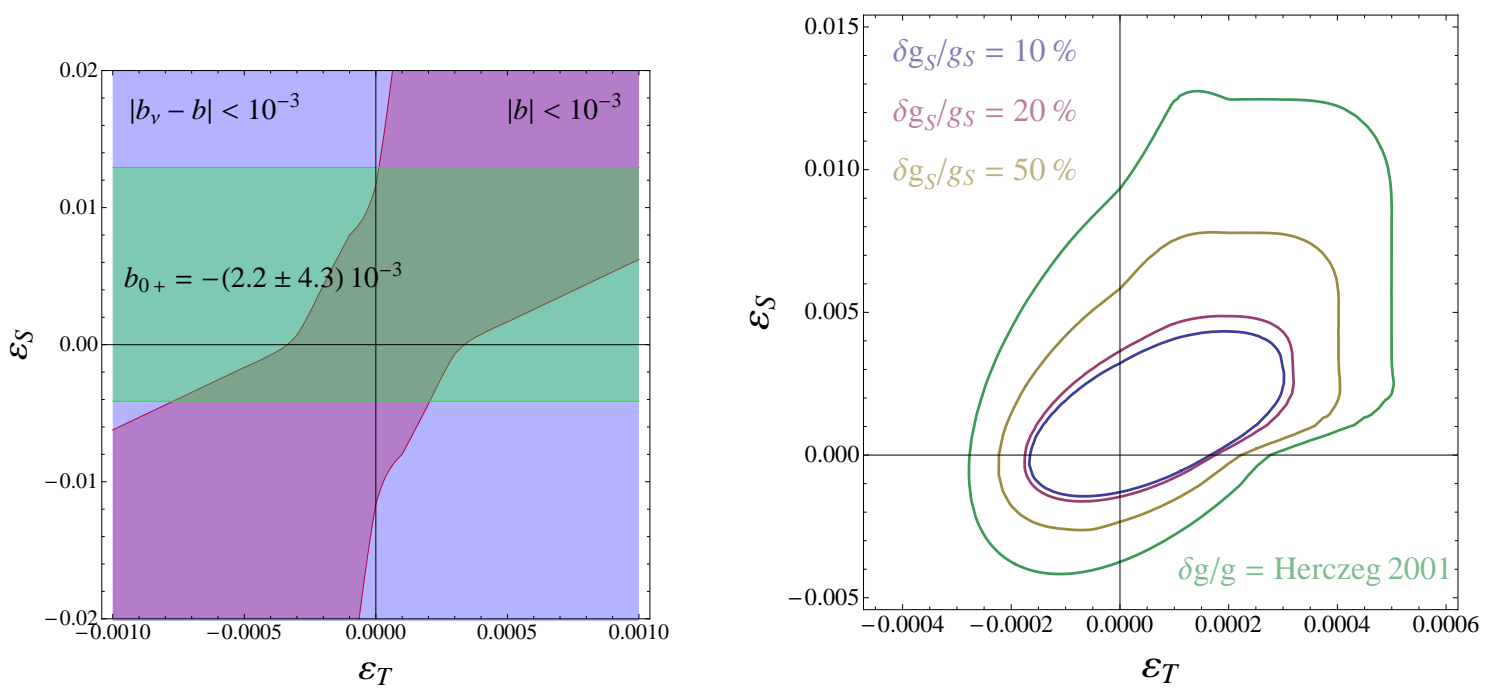

Figure 1: (Left) $90 \%$ confidence level (CL) allowed regions in the $\varepsilon_{S^{-}} \varepsilon_{T}$ plane implied by (i) the existing bound on $b_{0^{+}}$characterizing the $0^{+} \rightarrow 0^{+}$nuclear beta decays (green horizontal band); (ii) projected measurements of $b$ and $b_{v}-b$ in neutron decay (red bow-tie shapes and blue regions) at the $10^{-3}$ level; (iii) hadronic matrix elements taken in the ranges $0.25<g_{S}<1.0,0.6<g_{T}<2.3$ [8]. (Right) Combined 90\% $\mathrm{CL}$ allowed regions in the $\varepsilon_{S^{-}} \varepsilon_{T}$ plane based on existing limits on $b_{0^{+}}$from $0^{+} \rightarrow 0^{+}$nuclear beta decays and future neutron-decay measurements with projected sensitivity of $10^{-3}$ in $b$ and $b_{v}-b$. The four curves correspond to four different scenarios for the hadronic matrix elements: $0.25<g_{S}<1.0,0.6<g_{T}<2.3$ as quoted in Ref. [8]; lattice results with current central values from Ref. [12]) and $\delta g_{S} / g_{S}=50 \%, 20 \%, 10 \%$ with $\delta g_{T} / g_{T}=2 / 3 \delta g_{S} / g_{S}$ (this choice assumes that the ratio of fractional uncertainties in $g_{S}$ and $g_{T}$ will remain approximately constant as these uncertainties decrease). The effective couplings $\varepsilon_{S, T}$ are defined in the $\overline{\mathrm{MS}}$ scheme at $2 \mathrm{GeV}$. Note that the decrease in the allowed region becomes small for $\delta g_{S} / g_{S}<20 \%$, below which the constraints are dominated by the experimental uncertainty. 
figures, $b_{0^{+}}$represents the constraint from $0^{+} \rightarrow 0^{+}$nuclear beta decays. While the LHC will be able to produce $W^{\mathrm{BSM}}$ with masses in the $0.2-3-\mathrm{TeV}$ range and may provide precise measurements of their masses, the proposed neutron experiments will provide the most precise measurement of their spins and couplings, needed to reconstruct the TeV-scale theory that will replace the SM. Moreover, even if $W^{\mathrm{BSM}}$ is out of kinematic reach at the LHC (couplings are $O\left(10^{-4}\right)$ or smaller) or swamped by background, the interactions that it generates may lead to observable effects in the proposed neutron-decay experiments.

The right-hand-side of Fig. 1 shows that to keep the theoretical uncertainty on the reconstructed couplings $\varepsilon_{i}^{\text {BSM }}$ below the expected experimental error (estimated at $20 \%$, assuming a signal at the level of $10^{-3}$ ), a precision of $10-20 \%$ is required for the matrix elements $g_{S}$ and $g_{T}$.

We see, therefore, that a key ingredient needed to connect theory to experiments is the precise determination of the hadronic matrix elements of quark bilinear operators between the initial neutron and final proton states; that is, $g_{S, T}$. Since the initial and final states involve quarks at $\mathrm{GeV}$ scale, these matrix elements receive quantum corrections from the strongly interacting sector of the $\mathrm{SM}, \mathrm{QCD}$, irrespective of the TeV-scale theory. These corrections are intrinsically nonperturbative because the QCD coupling constant that enters into these calculations is of order unity. Lattice QCD (LQCD), a first-principles nonperturbative approach, provides the best known method for calculating these matrix elements. For the rest of the proceeding, we will introduce the concepts of lattice QCD for a general computational science background, followed by the methods used to control the systematic uncertainty in the lattice calculations and a discussion of how our $g_{S, T}$ impacts new-particle mass constraints.

\section{QCD and the Lattice}

QCD is the theory of the physics that dominates at very small scales, the femtoscale $\left(10^{-15} \mathrm{~m}\right)$, where fundamental particles (quarks and gluons) are bound inside the building blocks of matter, protons and neutrons. This is far smaller than the size of the atoms $\left(10^{-10} \mathrm{~m}\right)$ which form molecules (nanoscale, $10^{-9}$ ) in the materials we are familiar with. What do we learn by considering physics at this tiny scale? For one, we can understand how fundamental particles make up the proton and neutron, and more exotic particles such as hyperons. Further, we may try to see how they interact with each other. At the nuclear level, we can learn how the residual strong force binds nucleons together into the nuclei that form the cores of all atoms, that power the stars and perhaps one day fusion power plants. An understanding of how nuclear structure arises from fundamental physics may hold the key to the question of fine tuning (a mystery relevant to any carbon-based life forms). The interactions between hadrons at small scale also impact objects at the astrophysical scale, such as neutron stars. The strengths of these interactions can be input into descriptions of nuclear matter in simulations of the conditions in the center of neutron stars, where incredible pressure and density could allow exotic forms of matter to exist.

Quantum chromodynamics is the theory of the color force. It describes the strong interactions between quarks and gluons using an SU(3) gauge theory. Given a QCD action $S$ and interesting observables whose properties are described by an operator $\mathscr{O}$, one can compute the physical quantities of interest using a path integral (integrating over all possible configurations of gluonic and 

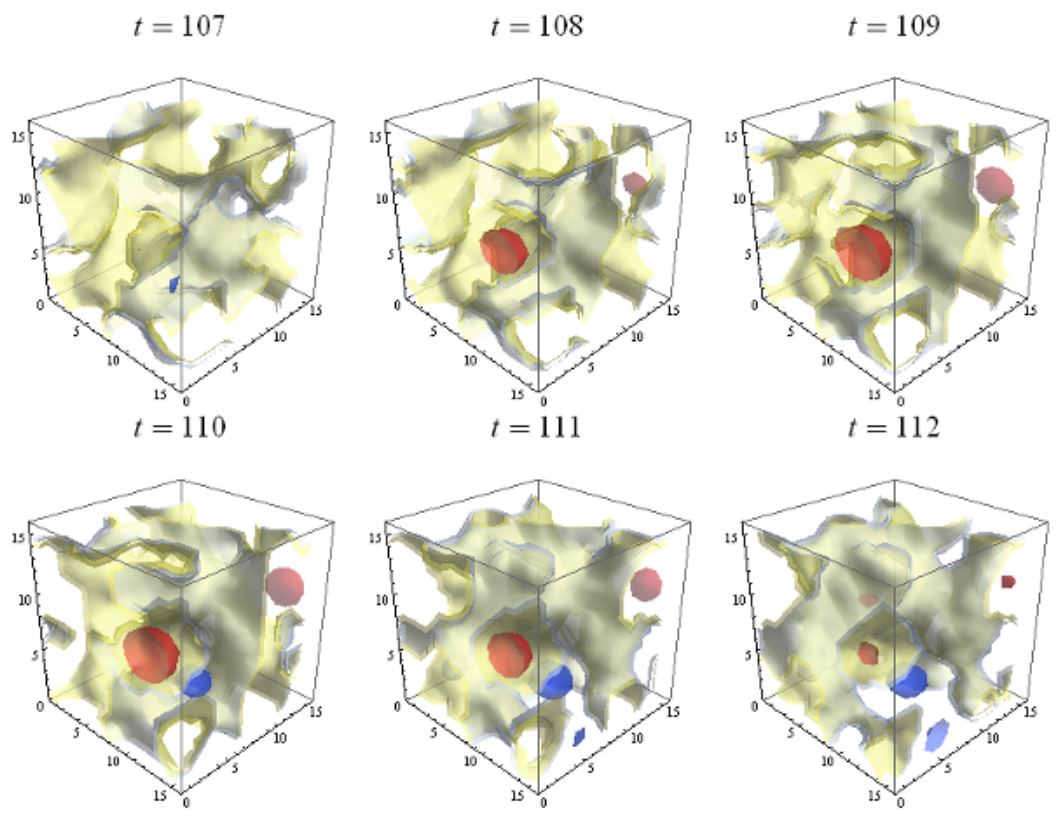

Figure 2: A selection of timeslices showing the topological charge of the vacuum calculated on one gaugefield configuration on a lattice with spacing approximately $0.1227 \times 10^{-15}$ meters with 16 lattice sites in each spatial direction. The evolution of the vacuum shown in this image occurs over $5.8 \times 10^{-25}$ seconds. The red regions correspond to instantons with positive topological winding number, while the blue correspond to instantons with negative winding number.

fermionic fields $A, \psi, \bar{\psi})$ :

$$
\langle\mathscr{O}[A, \psi, \bar{\psi}]\rangle=\frac{1}{Z} \int D A D \psi D \bar{\psi} e^{i S[A, \psi, \bar{\psi}]} \mathscr{O}[A, \psi, \bar{\psi}] .
$$

One of the interesting properties of quantum chromodynamics is confinement. That is, we never see free quarks in nature; rather, all we see are composite particles containing quarks, called hadrons, such as the lightest hadron, the pion, and the proton and neutron of ordinary nuclei. At large energy, the QCD coupling is small. We can simply make an expansion in terms of the coupling, and it converges well. At low energy, however, where the strong coupling becomes large, the theory becomes nonperturbative. Even just the vacuum of QCD is incredibly complicated; the QCD vacuum teems with topological charge and the results of the broken quark chiral symmetry. Figure 2 shows a few timeslices of the spatial distribution of topological charge in an example QCD vacuum configuration. Unlike the classical vacuum, to describe this is a task that is nearly impossible analytically. Therefore, nonperturbative approaches are essential to exploring QCD physics at this energy scale.

In order to study the interesting physics in the low-energy region, in the 1970s Kenneth Wilson proposed to discretize space and time in the path integral and to work in Euclidean space. Thus, lattice QCD (LQCD) was born. It contains two scales that are absent in continuum QCD, one ultraviolet (the lattice spacing $a$ ) and one infrared (the spatial extent of the box $L$ ). The problem now only involves a finite number of degrees of freedom and can be put on a computer for numerical integration. 

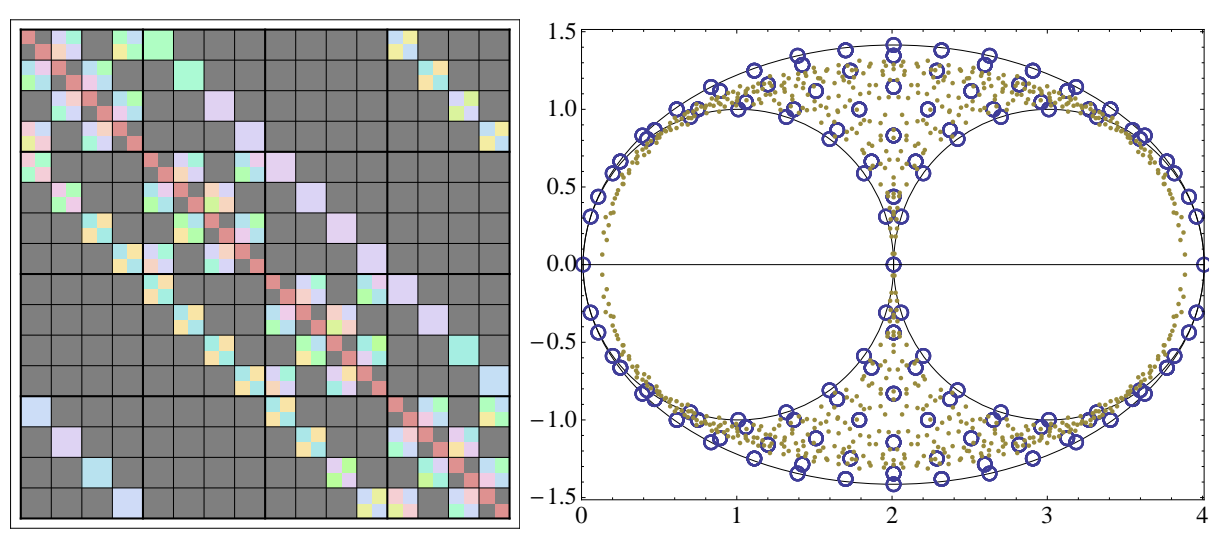

Figure 3: (left) Two-dimensional illustration of the spin structure of the Wilson Dirac operator. Gray is zero; blue is real and positive; red is negative; magenta and green are positive and negative imaginary. (right) Spectral plot for a two-dimensional Wilson Dirac operator. The blue circles indicate the results from free-field, while the olive dots have $\mathrm{U}(1)$ interacting gauge fields. The smaller the $m_{q}$, the worse the matrix condition number.

Of course, if computational resources were not an issue, we could solve all the QCD problems by brute force as long as we could write down the path integrals. However, it is not possible within a human lifetime to do so, so we have to examine the problem and solve it more cleverly. The number of degrees of freedom for a fermion here is the spatial volume times the number (3) of colors, times the number (4) of spins. The number of spatial points varies, for example, a lattice volume with $64^{3} \times 96$ has rank $300 \mathrm{M}$, and it cannot be fit onto a few workstations; a computational cluster with thousands of cores is necessary. Lattice practitioners use systems ranging from smallsize clusters hosted at universities to national supercomputer centers, such as Hopper at NERSC, or CPU-GPU hybrid systems, such as Keeneland at XSEDE.

In most cases, we spend the majority of computational hours inverting the Dirac operator. Due to the breaking of continuous rotation symmetry, multiple versions of the Dirac operator can be written on the lattice that describe the same physics in the continuum. Such a discrete Dirac operator is a sparse and structured matrix, properties that we can take advantage of to develop smarter solvers for our problem. To illustrate structure of our operator, we show a 2-dimensional version with $\mathrm{U}(1)$ gauge fields (in reality, we use a much more complicated $4 \mathrm{D}, \mathrm{SU}(3)$ theory); this particular form is the Wilson Dirac operator:

$$
D_{x, x^{\prime}}^{\mathrm{Wilson}}=(m+d) \delta_{x, x^{\prime}}-\sum_{\mu} \frac{1}{2}\left[\left(1+\gamma_{\mu}\right) U_{x, \mu} \delta_{x+\hat{\mu}, x^{\prime}}+\left(1-\gamma_{\mu}\right) U_{x, \mu}^{\dagger} \delta_{x, x^{\prime}+\hat{\mu}}\right]
$$

The left-hand side of Fig. 3 shows the spin structure of the two-dimensional version of Eq. 2.2. We can see that it is highly structured along diagonals, with nonzero terms in regular places, but mostly zero. The eigenvalues of this operator in the complex plane are shown on the right-hand side of Fig. 3. When interactions are turned off, the eigenvalues are these blue circles, while the olive points are the eigenvalues with thermalized U(1) gauge fields included. As the quark mass is decreased, entire eigenspectrum is shifted to the left; thus, the lowest eigenvalues approach zero and the matrix condition number diverges. This means that our inversion algorithms will run very 
slowly. Only recently have ensembles with physical pion masses become available; most of the time, we do the calculation with multiple heavier masses, and a procedure for extrapolation is necessary. Taking advantage of the structure of the operator, many techniques have been developed to solve the Dirac equation faster: eigenvector deflation, multi-mass solvers, multigrid acceleration, stochastic sources. Some such techniques have been examining the memory/communication problem, which will be needed to run QCD effectively on systems including GPUs [13, 14, 15].

Once we have $D^{-1}$, we can use it to calculate Green functions from which we will extract physical quantities. For example, we can calculate the pion mass by creating a pion at a certain point in space and time and annihilating it at another. In practice, we "contract" the inverse Dirac matrix with some spin/color matrix:

$$
C_{\pi}(t)=\langle\pi(0) \mid \pi(t)\rangle=\left\langle\bar{u} \gamma_{5} d(0) \bar{d} \gamma_{5} u(t)\right\rangle=\left\langle D^{-1}(0, t)\left(D^{-1}(0, t)\right)^{\dagger}\right\rangle .
$$

After momentum projection to the rest frame, the resulting Green function declines exponentially in the time direction with exponent equal to the mass. A similar process can be used in calculating nucleon matrix elements with nucleon interpolation operators and various insertion operators. Details are given in the upcoming section.

\section{Precision Neutron-Decay Matrix Elements}

The QCD vacuum we choose is $N_{f}=2+1+1$ flavors of highly improved staggered quarks (HISQ) [16, 17, 18, 19, 20, 21] generated by the MILC Collaboration [22]. Staggered-type fermions are notorious for their complications in calculations involving baryons, especially of matrix elements. Therefore, we use clover $(O(a)$-improved Wilson) fermion action in the valence sector for our calculation of nucleon matrix elements. We use hypercubic (HYP) smearing [23] of the gauge links before inverting the clover Dirac matrix needed to construct correlation functions [24, 25]. Using gauge fields averaged over a hypercube reduces short-distance noise (lattice artifacts) without changing long-distance physics. One advantage of HYP smearing is that the renormalization constants are close to the tree-level value, unity, as shown in Ref. [26].

To calculate the nucleon matrix elements (which lead to the $g_{S, T}$ we need for constraining the $\left.\varepsilon_{S, T}\right)$, we first calculate the matrix element of general form $\left\langle\chi^{N}\left(\vec{p}_{f}\right)\left|O_{\Gamma}\right| \chi^{N}\left(\vec{p}_{i}\right)\right\rangle$, where $O_{\Gamma}$ is $V_{\mu}=$ $\bar{u} \gamma_{\mu} d$ for the isovector vector current, $A_{\mu}=\bar{u} \gamma_{\mu} \gamma_{5} d$ for isovector axial current, etc., and $\vec{p}_{\{i, f\}}$ are the initial and final nucleon momenta. Such a matrix element is extracted from an appropriate threepoint correlation function after Fourier transforming out the spatial dependence and projecting on the baryonic spin, leaving a time-dependent three-point correlator of the form

$$
\mathscr{C}_{\Gamma}^{(3), T}\left(t_{i}, t, t_{f} ; \vec{p}_{i}, \vec{p}_{f}\right)=Z_{\Gamma} \sum_{n, n^{\prime}} f_{n, n^{\prime}} \sum_{s, s^{\prime}} T_{\alpha \beta} u_{n^{\prime}}^{\beta}\left(\vec{p}_{f}, s^{\prime}\right) \times\left\langle N_{n^{\prime}}\left(\vec{p}_{f}, s^{\prime}\right)\left|O_{\Gamma}\right| N_{n}\left(\vec{p}_{i}, s\right)\right\rangle \bar{u}_{n}^{\alpha}\left(\vec{p}_{i}, s\right),
$$

where $f_{n, n^{\prime}}$ contains kinematic factors involving the energies $E_{n}$ and amplitudes $\mathscr{A}_{n}$ between the creation and annihilation operators and the corresponding states. The latter are obtained from analysis of the two-point correlators with $n$ and $n^{\prime}$ labeling the different energy states (starting from $0) . Z_{\Gamma}$ is the operator renormalization, which is determined nonperturbatively. The projection $T$ used here is $T_{\text {mix }}=\frac{1}{4}\left(1+\gamma_{4}\right)\left(1+i \gamma_{5} \gamma_{3}\right)$. In this work we are interested in only the ground-state matrix element with $n=n^{\prime}=0$. 


\begin{tabular}{ccccccc}
\hline \hline Ensemble & $a(\mathrm{fm})$ & $M_{\pi}(\mathrm{MeV})$ & $L^{3} \times T$ & $M_{\pi} L$ & $a t_{\text {sep }}$ & $N_{\text {Measurement }}$ \\
\hline a12m310 & 0.12 & 310 & $24^{3} \times 64$ & 4.5 & $\{8,9,10,11,12\}$ & $4052^{*}$ \\
a12m220S & 0.12 & 220 & $24^{3} \times 64$ & 3.2 & 10 & 12000 \\
a12m220 & 0.12 & 220 & $32^{3} \times 64$ & 4.4 & $\{8,10,12\}$ & $3832^{*}$ \\
a12m220L & 0.12 & 220 & $40^{3} \times 64$ & 5.4 & 10 & 8000 \\
a09m310 & 0.09 & 310 & $32^{3} \times 96$ & 4.5 & $\{10,12,14\}$ & 7048 \\
a09m220 & 0.09 & 220 & $48^{3} \times 96$ & 4.8 & $\{10,12,14\}$ & $3560^{*}$ \\
a09m130 & 0.09 & 130 & $64^{3} \times 96$ & 3.8 & $\{10,12,14\}$ & 3532 \\
\hline \hline
\end{tabular}

Table 1: The ensembles used to analyze the tensor and scalar charges. “*” indicates the statistics are continuing to increase at the time of this report.

The usual suspects for the systematics of a lattice measurement are renormalization, lattice discretization artifacts, excited-state contamination, unexpected chiral behavior and finite-volume corrections. In our case, we have performed nonperturbative renormalization for all of our operators; however, we do not have $O(a)$-improved operators, and our plan is to perform calculations at three lattice spacings to study and remove the discretization effects. From our current study on the 0.09-fm and 0.12-fm lattices, we find such effects to be insignificant within our current statistical uncertainty. Chiral extrapolation to physical pion mass can also introduce big systematics; luckily, we have one ensemble with pion masses as low as $130 \mathrm{MeV}$; thus, we are able to interpolate our matrix elements rather than extrapolate. This gives us better control over this systematic.

Last but not least, in the following subsections, we discuss in greater detail the excited-state contamination and finite-volume corrections. We find that these are more significant than what others have observed in the past.

\subsection{Excited-State Contamination}

One of the notorious systematics in extracting precision neutron-decay matrix elements is excited-state contamination, and many lattice-QCD calculations have been trying hard to address this issue. There are two possible ways to reduce contributions from excited states: by reducing the overlap of the interpolating operator with the excited states and by increasing the time separation $t_{\text {sep }}=t_{f}-t_{i}$ between the source and sink to exponentially suppress excited-state contamination. However, the signal-to-noise ratios worsen as one increases $t_{\text {sep }}$; thus, a careful analysis extracting ground-state nucleon matrix elements with limited size of $t_{\text {sep }}$ is important.

To deal with this systematic, we consider the excited-state mass $M_{1}$ and its coupling to our operator with amplitude $\mathscr{A}_{1}$. We can write the three-point function with source shifted to $t_{i}=0$, operator insertion at $t=t$ and sink at $t_{f}=t_{\text {sep }}$ as

$$
\begin{aligned}
\mathscr{C}_{\Gamma}^{(3), T}\left(t_{i}, t, t_{f} ; \vec{p}_{i}, \vec{p}_{f}\right) \approx\left|\mathscr{A}_{0}\right|^{2}\left\langle 0\left|O_{\Gamma}\right| 0\right\rangle e^{-M_{0}\left(t_{f}-t_{i}\right)}+\left|\mathscr{A}_{1}\right|^{2}\left\langle 1\left|O_{\Gamma}\right| 1\right\rangle e^{-M_{1}\left(t_{f}-t_{i}\right)}+ \\
\mathscr{A}_{0} \mathscr{A}_{1}^{*}\left\langle 0\left|O_{\Gamma}\right| 1\right\rangle e^{-M_{0}\left(t-t_{i}\right)} e^{-M_{1}\left(t_{f}-t\right)}+\mathscr{A}_{0}^{*} \mathscr{A}_{1}\left\langle 1\left|O_{\Gamma}\right| 0\right\rangle e^{-M_{1}\left(t-t_{i}\right)} e^{-M_{0}\left(t_{f}-t\right)},
\end{aligned}
$$

where $\left\langle n^{\prime}\left|O_{\Gamma}\right| n\right\rangle$ is an abbreviation for $\left\langle N_{n^{\prime}}\left(\vec{p}_{f}, s^{\prime}\right)\left|O_{\Gamma}\right| N_{n}\left(\vec{p}_{i}, s\right)\right\rangle$. To extract $\left\langle 0\left|O_{\Gamma}\right| 0\right\rangle$ from the twoand three-point functions, we make the following different kinds of fits. In each case, we apply a 

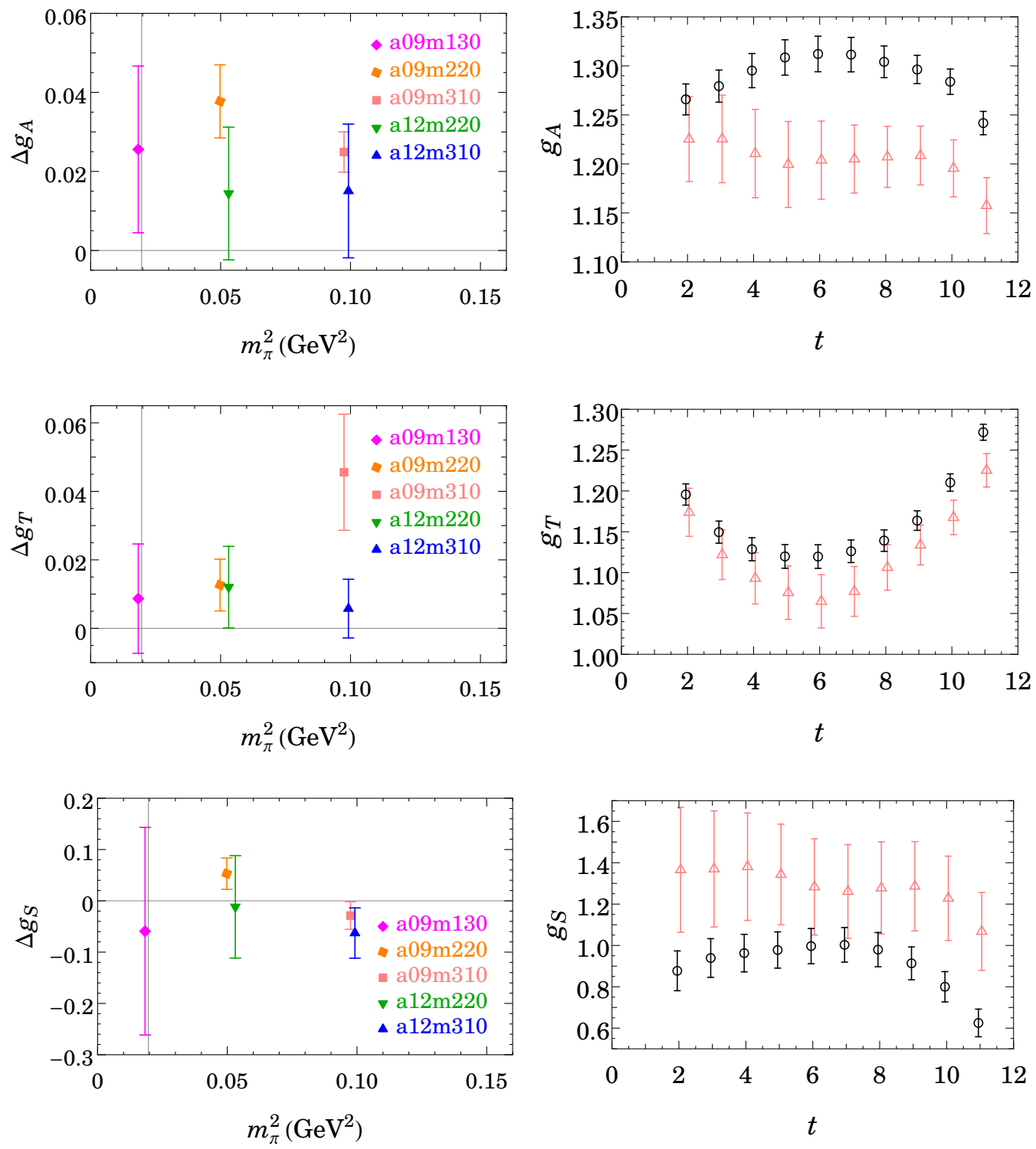

Figure 4: (Left column)The jackknifed difference in extracted charges due to ignoring the "RR" term, $\left\langle 1\left|O_{\Gamma}\right| 1\right\rangle$, when simultaneously fitting all the $t_{\text {sep }}$ three-point correlators for the axial, tensor, and scalar charges (from top to bottom) as functions of $m_{\pi}^{2}$. The detailed parameters associated with the ensemble labels can be found in Table 1. (Right column) Finite volume effects on axial, tensor and scalar charges (from top to bottom); black circles and pink triangles correspond to $m_{\pi} L=3.2$ and 5.3, respectively. 
nonlinear least-square fitter that automatically selects a fit range appropriate to the form used. For each form on each correlator, the fit range is expanded as long as the quality of the fit (in terms of uncorrelated $\chi^{2} /$ dof) does not sharply decline.

In Ref. [26] where we concentrate on our results from $a=0.12 \mathrm{fm}$, we found using multiple $t_{\text {sep }}$ ranging from 0.96 to $1.44 \mathrm{fm}$, our 2-2 $\operatorname{sim}_{\text {method }}{ }^{1}$ and 2-2 $R R$ method ${ }^{2}$ are consistent; i.e. the jackknifed difference in extracting ground-state charges are consistent with zero. However, as we move to smaller lattice spacing, $a=0.09 \mathrm{fm}$, the difference becomes significant. This is not too surprising, since the mass difference between the excited and ground state becomes smaller in lattice units as lattice spacing $a$ gets smaller; thus, it is important to include the higher-order terms that people do not usually include. This also indicates the exploratory study of $t_{\mathrm{sep}}$ done at coarser lattice spacing may not be useful any more.

The left column of Fig. 4 shows the jackknifed difference between including the "RR" term (the excited-excited matrix element, $\left\langle 1\left|O_{\Gamma}\right| 1\right\rangle$ ) and the one without (only including one leading matrix element; note that the popular "summation" method implicitly uses this assumption) for axial, tensor and scalar charges. The effect is most evident for the axial charge and not as clear in the tensor and scalar charges with our current statistics; our upcoming data may help to clarify the trend.

\subsection{Finite-Volume Effects}

Unfortunately, there are not many studies nor effective-theory guides for the finite-volume effects on the tensor and scalar charges. The most well studied case for this systematic is $g_{A}$. Therefore, we use $g_{A}$ as a guide and estimate our finite-volume systematics for the other charges in similar fashion.

Finite-volume effects are known to decrease the value of $g_{A}$ by a significant amount in lattice calculations. Two commonly used formulations to determine finite-volume corrections are HBChPT [27] and its variation SSE [28]. Groups that estimate their finite-volume corrections using the Refs. [27, 28] have reported small systematics, less than $1 \%$ for $m_{\pi} L \approx 3$. However, an earlier quenched RBCK study [29] saw more significant effects, as shown in Refs. [27, 28]. Recent RBC/UKQCD $N_{f}=2+1$ studies also see a significant central-value shift of about 0.05 and 0.12 with pion masses 670 and $420 \mathrm{MeV}$ when the volume changes from 2.74 to $1.82 \mathrm{fm}$ (which corresponds to $m_{\pi} L$ going from 9.3 to 6.2 and 5.3 to 3.9 , respectively). QCDSF has multiple lattice spacings ( $a \in[0.06,0.078] \mathrm{fm}$ ) with various pion masses and volumes, and also sees a bigger volume correction than suggested in Refs. [27, 28].

We carry out a detailed finite-volume study at the $220-\mathrm{MeV}$ pion mass with lattice spacing $0.12 \mathrm{fm}$; this means that $m_{\pi} L$ ranges from 3.2 to 5.3. When we just look at the three-point/twopoint correlator ratios (a plateau fit to the middle time-insertion points would give unnormalized charges), as shown in the right column of Fig. 4, we found a bigger increase in $g_{A}$ when one approaches large volume, but the difference in $g_{S, T}$ becomes less clear within the statistical errors.

\footnotetext{
${ }^{1}$ We use $\mathscr{A}_{0}, \mathscr{A}_{1}, M_{0}$ and $M_{1}$ extracted from a fit to the two-point function, and simultaneously fit to all $t_{\text {sep }}$ threepoint functions. These amplitudes and masses are used in a two-parameter fit to the three-point function to estimate $\left\langle 0\left|O_{\Gamma}\right| 0\right\rangle$ and $\left\langle 1\left|O_{\Gamma}\right| 0\right\rangle$. In the case of charges where both initial and final nucleon operators are at rest, we can assume $\left\langle 0\left|O_{\Gamma}\right| 1\right\rangle$ and $\left\langle 1\left|O_{\Gamma}\right| 0\right\rangle$ are equal, and we analyze only the real part of the three-point function.

${ }^{2}$ Similar to $2-2 \mathrm{sim}$ method, but we keep terms up to $\left\langle 1\left|O_{\Gamma}\right| 1\right\rangle$ in the three-point function.
} 
Our strategy is to analyze these charges and model in the finite-volume correction terms that best describe our data.

\subsection{Continuum Extrapolation}

We extrapolate all the charges to the continuum limit, i.e. $m_{\pi}=m_{\pi}^{\text {phys }}$ and $V \rightarrow \infty$, as shown in Fig. 5, using a simple ansatz such as linear $m_{\pi}^{2}$ for mass dependence and $e^{-m_{\pi} L}$ for volume. With our current statistics, there is no significant dependence on the lattice spacing; thus, we ignore that dependence for now. In the future, we plan to include a third lattice spacing, $0.06 \mathrm{fm}$, and perform a proper extrapolation.

We first look at the axial charge, which has a firm experimental measurement. Such an exercise can give us an indication whether our simple ansatz is reasonable so that we can proceed to the less known cases of the tensor and scalar charges. Our simultaneous fit to $g_{A}$ gives 1.26(3), which is consistent with the latest PDG average experimental value, 1.2701(25). Although our lightest pion mass is $130 \mathrm{MeV}$, its volume is not big enough (whose $m_{\pi} L$ is only 3.8); as we see in the previous subsection on the finite-volume systematics, it requires finite-volume correction. Thus, a simultaneous fit is necessary to extract the correct value.

Given the encouraging results from the axial charge, we proceed to the tensor and scalar charges. The volume and mass dependence of the tensor charge is much milder than axial case, and our preliminary result gives 1.03(4). On the other hand, given the relatively noisier signal in the scalar charge, it is harder to see a clear trend; our current analysis give 0.86(13) at the continuum limit. Future improvement of the statistics, especially on the $130-\mathrm{MeV}$ ensemble would help to reduce the error.

\section{Impact on New-Physics Searches}

Finally, we can combine the tensor and scalar charges with experimental data to determine the allowed region of the parameter space for scalar and tensor BSM couplings (denoted $\varepsilon$ ). We can combine the current knowledge of $g_{S, T}$ and the existing experimental data for the nuclear beta decay $0^{+} \rightarrow 0^{+}$transition and others, such as $\beta$ symmetry in Gamow-Teller ${ }^{60} \mathrm{Co}$, longitudinal polarization ratio between Fermi and Gamow-Teller transitions in ${ }^{114} \mathrm{In}$, positron polarization in polarized ${ }^{107}$ In and beta-neutrino correlation parameters in nuclear transitions. Using the $g_{S, T}$ from the model estimations and combining with the existing nuclear experimental data, we get the constraints shown the outermost band in Fig. 6. Simply replacing the model estimations of $g_{S, T}$ with our present lattice-QCD values of the scalar and tensor charges, we find the constraints on $\varepsilon_{S, T}$ are greatly improved as shown in the middle contour in Fig. 6. There are ongoing and planned ultracold neutron (UCN) experiments studying neutron beta decay to look for deviations from the SM in the Fierz term and the neutrino asymmetry parameter ( $b$ and $b_{v}$ )to the level of $10^{-3}$ by $\mathrm{UCNb} / \mathrm{B}$ experiments at LANL or Nab at ORNL. Experiments taking advantage of the high-intensity source of ${ }^{6} \mathrm{He}$ at CEPTA at University of Washington can also probe the tensor current [31] through pure Gamow-Teller decay. Combining the expected data and existing measurements, and with lattice inputs of $g_{S, T}$, we see the uncertainties in $\varepsilon_{S, T}$ are significantly improved, as shown as the innermost region. This shows the importance of the precision experimental inputs in combination with theo- 

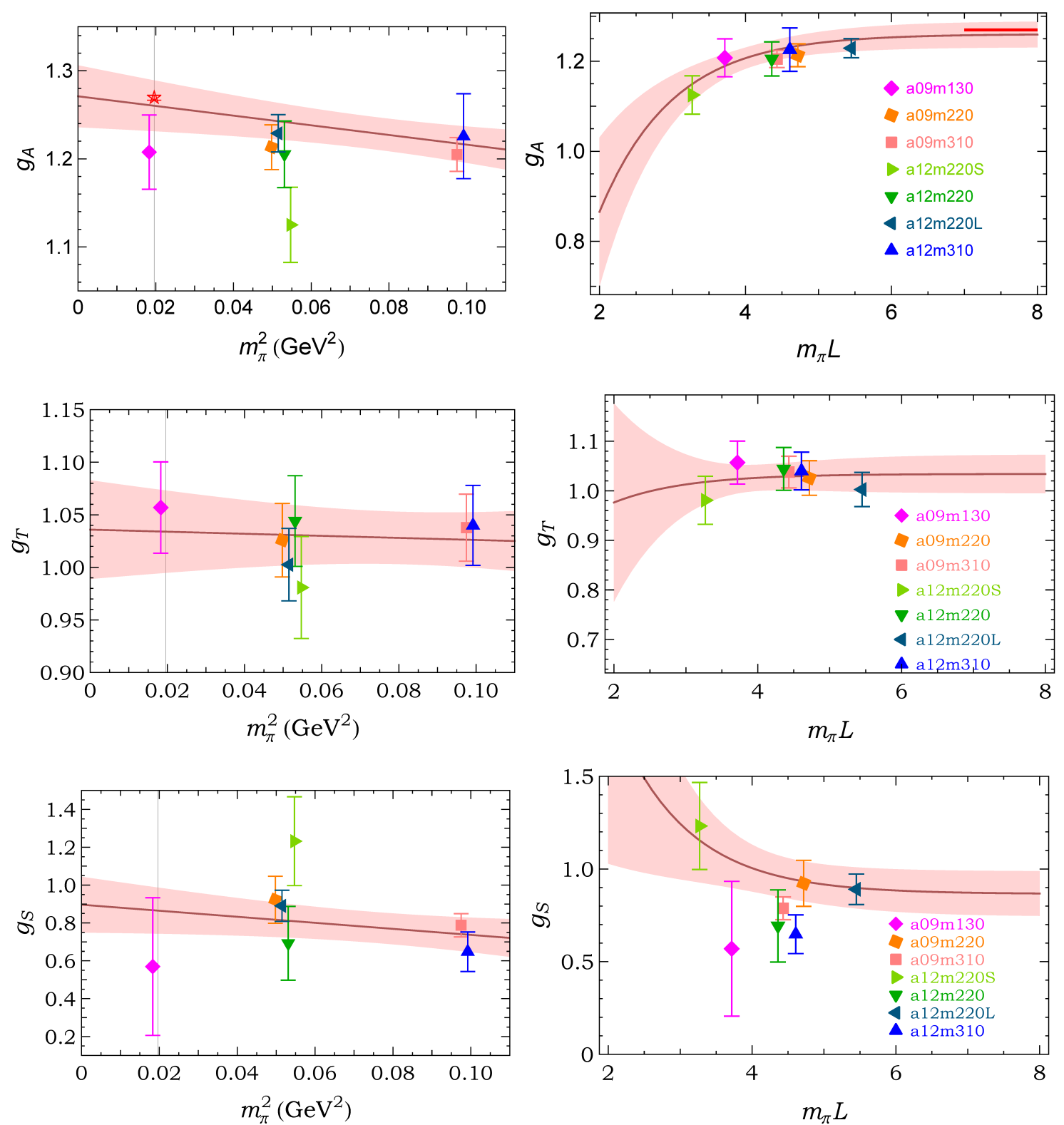

Figure 5: Simultaneous extrapolation of axial (top row), tensor (middle row) and scalar (bottom row) charges to continuum limit, $m_{\pi}=m_{\pi}^{\text {phys }}$ (left column) and $V \rightarrow \infty$ (right column). The detailed parameters associated with the ensemble labels can be found in Table 1. In the case of axial charge, the red star and horizontal line mark the PDG averaged experimental value. The bands in the plots have been projected to $V \rightarrow \infty$ (for left column) and $m_{\pi}=m_{\pi}^{\text {phys }}$ (right column). 


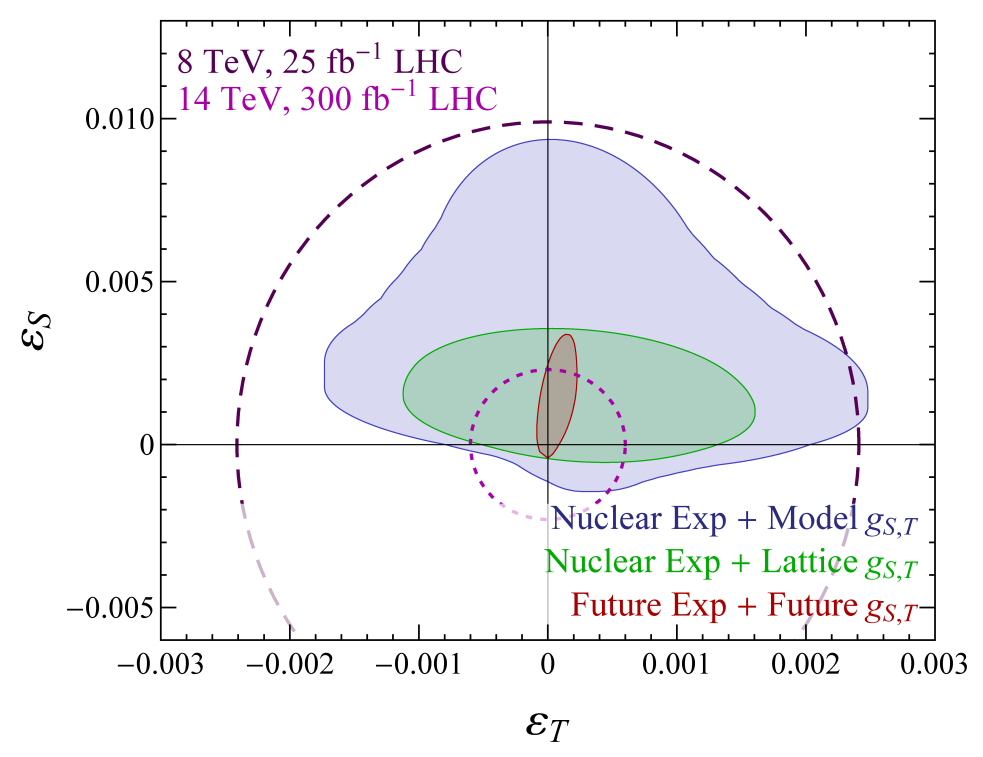

Figure 6: $\varepsilon_{S^{-}} \varepsilon_{T}$ allowed parameter region using different experimental and theoretical inputs as discussed in the text. All estimates are in the $\overline{\mathrm{MS}}$ scheme at $2 \mathrm{GeV}$. (left) The constraints from low-energy nuclear experiments and model estimates for $g_{S, T}$ are shown by the outer blue region; and the improvement on using lattice estimates of $g_{S, T}$ given in this paper reduce this to the middle green region. The inner red region indicates future bounds assuming UCN experiments provide $\left|b_{v}-b\right|<10^{-3}$ and $|b|<10^{-3},{ }^{6} \mathrm{He}$ experiments provide precision bounds, and errors in $g_{S}$ lattice estimates is reduced to $10 \%$. The constraint from radiative pion decay is shown by the two vertical lines. The low-energy constraints are compared to those from the LHC. The dashed purple and inner dotted magenta lines are the near-term and future longterm expectations using background inputs from Ref. [30].

retical advances. These upper bounds on the effective couplings $\varepsilon_{S, T}$ correspond to lower bounds for the scales $\Lambda_{S, T}$ at 5.6 and $10.3 \mathrm{TeV}$, respectively, for new physics in these channels.

How do the constraints from high-energy experiments compare? We can estimate the $\varepsilon_{S, T}$ constraints from LHC current bounds and near-term expectations through effective Lagrangian

$$
\mathscr{L}=-\frac{\eta_{S}}{\Lambda_{S}^{2}} V_{u d}(\bar{u} d)\left(\bar{e} P_{L} v_{e}\right)-\frac{\eta_{T}}{\Lambda_{T}^{2}} V_{u d}\left(\bar{u} \sigma^{\mu v} d\right)\left(\bar{e} \sigma_{\mu v} P_{L} v_{e}\right)
$$

where $\eta_{S, T}= \pm 1$. and $\Lambda_{S, T}$ are connected to $\varepsilon_{S, T}$ through vacuum expectation values. Using the tail of the transverse-mass distribution in the reaction $p p \rightarrow e \bar{v}+X$ (i.e. the region where $m_{T}>$ $m_{T}^{\text {cut }}$ ), we show three bounds from the LHC for different center-of-mass energies and integrated luminosity. The transverse-mass cut is chosen such that the expected Standard-Model background is less than one event. For the brown ellipse, the background is taken from the measured value at CMS [30]; otherwise, the background is estimated by computing at tree level the transverse-mass distribution due to the production of a high- $p_{T}$ lepton from an off-shell $W$. For further details of this analysis, refer to Refs. [12] and [32]. In Fig. 6 we make an illustrative comparison of the constraints on $\varepsilon_{S, T}$ (defined at $2 \mathrm{GeV}$ in the $\overline{\mathrm{MS}}$ scheme) projected limits from the LHC. The outer dashed purple ellipse gives the LHC expected constraint using the full current 8-TeV dataset; 
the inner dotted magenta ellipse gives the expected final LHC constraint with maximum lifetime luminosity at the $14-\mathrm{TeV}$ design energy.

If experiments can measure these BSM signals to be nonzero, combining with our precision $g_{S, T}$ calculations, we will be able to set a range of possible masses for potential new particles that may be probed directly by high-energy colliders, such as the LHC. If experiment cannot rule out the SM, then we will provide lower bounds for the scale of new physics in these channels, which will help to rule out certain classes of BSM models. As experimental precision on these quantities improve, it is important that the precision of these neglected lattice matrix elements do not become an obstacle to probing the precision frontier for new physics. We will accomplish this in the future by improving our calculation with physical pion masses, nonperturbative renormalization, improved statistics and continuum extrapolation.

\section{Acknowledgments}

HWL would like to thank the conference organizers for the financial support to make the trip. The calculations were performed using the Chroma software suite [33]. Computations for this work were carried out in part on facilities of the USQCD Collaboration, which are funded by the Office of Science of the U.S. Department of Energy. This work used the Extreme Science and Engineering Discovery Environment (XSEDE), which is supported by National Science Foundation grant number OCI-1053575. Some of the early parameter tunnng were initially done on Hyak clusters at the University of Washington managed by the UW Information Technology, using hardware awarded by NSF grant PHY-09227700. The speaker is supported by the DOE grant DE-FG02-97ER4014.

\section{References}

[1] A. Saunders, J. Anaya, T. Bowles, B. Filippone, P. Geltenbort, et al., Phys.Lett. B593, 55 (2004), nucl-ex/0312021.

[2] M. Anselmino, M. Boglione, U. D’Alesio, A. Kotzinian, F. Murgia, et al., Nucl.Phys.Proc.Suppl. 191, 98 (2009), 0812.4366.

[3] M. Diefenthaler (HERMES Collaboration) pp. 579-582 (2007), 0706.2242.

[4] R. Seidl et al. (Belle Collaboration), Phys.Rev. D78, 032011 (2008), 0805.2975.

[5] I. Cloet, W. Bentz, and A. W. Thomas, Phys.Lett. B659, 214 (2008), 0708.3246.

[6] M. Wakamatsu, Phys.Lett. B653, 398 (2007), 0705.2917.

[7] H.-x. He and X.-D. Ji, Phys.Rev. D52, 2960 (1995), hep-ph/9412235.

[8] P. Herczeg, Prog.Part.Nucl.Phys. 46, 413 (2001).

[9] S. L. Adler, J. Colglazier, E.W., J. Healy, I. Karliner, J. Lieberman, et al., Phys.Rev. D11, 3309 (1975).

[10] V. P. Gudkov, G. Greene, and J. Calarco, Phys.Rev. C73, 035501 (2006), nucl-th/0510012.

[11] N. Severijns, M. Beck, and O. Naviliat-Cuncic, Rev.Mod.Phys. 78, 991 (2006), nucl-ex/0605029.

[12] T. Bhattacharya, V. Cirigliano, S. D. Cohen, A. Filipuzzi, M. Gonzalez-Alonso, et al., Phys.Rev. D85, 054512 (2012), 1110.6448. 
[13] B. Joo (USQCD Collaboration, Hadron Spectrum Collaboration), J.Phys.Conf.Ser. 180, 012070 (2009).

[14] R. Babich, M. Clark, B. Joo, G. Shi, R. Brower, et al. (2011), 1109.2935.

[15] S. D. Cohen, R. Brower, M. Clark, and J. Osborn, PoS LATTICE2011, 030 (2011), 1205.2933.

[16] E. Follana et al. (HPQCD Collaboration, UKQCD Collaboration), Phys.Rev. D75, 054502 (2007), hep-lat/0610092.

[17] E. Follana et al. (HPQCD Collaboration, UKQCD Collaboration), Nucl.Phys.Proc.Suppl. 129\&130, 384 (2004), hep-lat/0406021.

[18] A. Bazavov et al. (MILC collaboration), Phys.Rev. D82, 074501 (2010), 1004.0342.

[19] A. Bazavov et al. (MILC Collaboration), PoS LAT2009, 123 (2009), 0911.0869.

[20] A. Bazavov et al. (MILC Collaboration), PoS LATTICE2008, 033 (2008), 0903.0874.

[21] A. Bazavov, D. Toussaint, C. Bernard, J. Laiho, C. DeTar, et al., Rev.Mod.Phys. 82, 1349 (2010), 0903.3598.

[22] A. Bazavov et al. (MILC Collaboration), Phys.Rev. D87(5), 054505 (2013), 1212.4768.

[23] A. Hasenfratz and F. Knechtli, Phys.Rev. D64, 034504 (2001), hep-lat/0103029.

[24] J. Bratt et al. (LHPC Collaboration), Phys.Rev. D82, 094502 (2010), 1001.3620.

[25] H.-W. Lin and K. Orginos, Phys.Rev. D79, 034507 (2009), 0712.1214.

[26] T. Bhattacharya, S. D. Cohen, R. Gupta, A. Joseph, and H.-W. Lin (2013), 1306.5435.

[27] S. R. Beane and M. J. Savage, Phys.Rev. D70, 074029 (2004), hep-ph/0404131.

[28] A. A. Khan, M. Gockeler, P. Hagler, T. Hemmert, R. Horsley, et al., Phys.Rev. D74, 094508 (2006), hep-lat/0603028.

[29] S. Sasaki, K. Orginos, S. Ohta, and T. Blum (RIKEN-BNL-Columbia-KEK Collaboration), Phys.Rev. D68, 054509 (2003), hep-lat/0306007.

[30] S. Chatrchyan et al. (CMS Collaboration), Phys.Rev. D87(7), 072005 (2013), 1302.2812.

[31] A. Knecht, Z. Alexander, Y. Bagdasarova, T. Cope, B. Delbridge, et al. (2012), 1208.6433.

[32] V. Cirigliano, M. Gonzalez-Alonso, and M. L. Graesser, JHEP 1302, 046 (2013), 1210.4553.

[33] R. G. Edwards and B. Joo (SciDAC Collaboration, LHPC Collaboration, UKQCD Collaboration), Nucl.Phys.Proc.Suppl. 140, 832 (2005), hep-lat/0409003. 